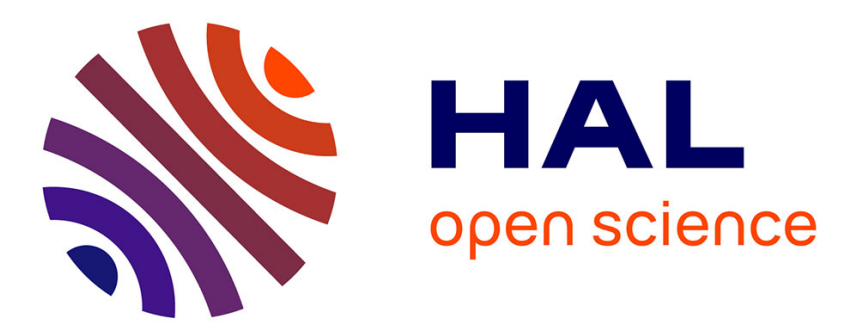

\title{
Generalized Spatial Modulation for Wireless Terabits Systems under sub-THz Channel with RF Impairments
}

Majed Saad, Faouzi Bader, Ali Al Ghouwayel, Hussein Hijazi, Nizar Bouhel, Jacques Palicot

\section{- To cite this version:}

Majed Saad, Faouzi Bader, Ali Al Ghouwayel, Hussein Hijazi, Nizar Bouhel, et al.. Generalized Spatial Modulation for Wireless Terabits Systems under sub- THz Channel with RF Impairments. IEEE International Conference on Acoustics, Speech and Signal Processing (ICASSP), May 2020, Barcelona, Spain. 10.1109/ICASSP40776.2020.9053208 . hal-02483233v2

\section{HAL Id: hal-02483233 \\ https://hal.science/hal-02483233v2}

Submitted on 27 Jun 2020

HAL is a multi-disciplinary open access archive for the deposit and dissemination of scientific research documents, whether they are published or not. The documents may come from teaching and research institutions in France or abroad, or from public or private research centers.
L'archive ouverte pluridisciplinaire HAL, est destinée au dépôt et à la diffusion de documents scientifiques de niveau recherche, publiés ou non, émanant des établissements d'enseignement et de recherche français ou étrangers, des laboratoires publics ou privés. 


\title{
GENERALIZED SPATIAL MODULATION FOR WIRELESS TERABITS SYSTEMS UNDER SUB-THZ CHANNEL WITH RF IMPAIRMENTS
}

\author{
Majed Saad ${ }^{\dagger}$, Faouzi Bader ${ }^{\dagger}$, Ali C. Al Ghouwayel ${ }^{\star}$, Hussein Hijazi $^{\star}$, Nizar Bouhel ${ }^{\dagger}$, Jacques Palicot ${ }^{\dagger}$ \\ † IETR/CentraleSupélec, Rennes Campus Cesson-Sévigné, France \\ $\ddagger$ Institut Supérieur d’Électronique de Paris, 75006 Paris, France \\ * School of Engineering, Lebanese International University LIU, Lebanon \\ $\dagger$ majed.saad@ieee.org, \{faouzi.bader, nizar.bouhel, jacques.palicot\}@centralesupelec.fr \\ * $\{$ ali.ghouwayel, hussein.hijazi $\} @$ liu.edu.lb
}

\begin{abstract}
Multiple-Input Multiple-Output (MIMO) technique with Index Modulation (IM) over sub-TeraHertz (sub-THz) bands represent a promising solution to design new wireless ultrahigh data rate systems. However, the system design over sub-THz bands suffers from many technological limitations and severe RF-impairments such as low output power, limited resolution of high-speed low-power Analog-to-Digital Converters and important Phase Noise (PN) introduced by the Local Oscillator (LO). In this paper, different modulations schemes with Generalized Spatial Modulation (GSM) are compared from different perspectives while considering the sub-THz impairments. The effect of PN has been investigated for these modulation schemes in sub- $\mathrm{THz}$ channels using uniform linear and rectangular antenna arrays. The obtained results reveal that QPSK-GSM system is the best combination compared to GSM systems with any other $M$ ary modulation scheme (e.g. PSK, DPSK, QAM, PAM). Compared to DQPSK-GSM and 4PAM-GSM at $12 \mathrm{bpcu}$, same number of receive and activated transmit antennas, the QPSK-GSM system offers a gain ranging from $3.4 \mathrm{~dB}$ up to $5 \mathrm{~dB}$. The results reveals that low to medium residual PN in distributed oscillator architecture can be tolerated when using GSM-QPSK without phase noise mitigation. Thus, enforcing the GSM to be a promising candidate for ultra-high wireless data rate communication in sub- $\mathrm{THz}$ bands.
\end{abstract}

Index Terms - Beyond 5G, Sub-Terahertz systems (sub$\mathrm{THz}$ ), Generalized Spatial Modulation (GSM), Phase Noise (PN), sub-Terahertz MIMO channel.

The research leading to these results received funding from the French National Research Agency under Grant Agreement no. ANR-17-CE25-0013 within the frame of the project BRAVE. We would like to thank CNRS GdRISIS for the research mobility grant, BRAVE project partners: SIRADEL for providing the sub-THz MIMO channels from their ray-based channel simulation and CEA-Leti for the suitable sub-THz phase noise model.

\section{INTRODUCTION}

Multiple-Input Multiple-Output (MIMO) system with Index Modulation (IM) as Generalized Spatial Modulation (GSM) [1] is a promising technique for wireless ultra-high data rates system where it achieves the best performance and lowest power consumption when using low order modulation schemes in correlated $\backslash$ uncorrelated Rayleigh and Rician channels [2]. However, GSM performance degrades in highly correlated channels and many enhancement techniques are proposed in [3] and [4] to reduce this degradation.

Moreover, millimeter wave $(\mathrm{mmW})$ and sub-TeraHertz (sub-THz) bands are considered for beyond 5G ultra-high data rate scenarios [5]. However, these bands especially the sub- $\mathrm{THz}$ suffers from higher channel attenuation and sensitivity to small environment details [6][7], severe RFimpairments and many technological limitations such as low output power, limited resolution of high-speed low-power Analog-to-Digital Converters (ADCs) [8], important Phase Noise (PN) introduced by the Local Oscillator (LO) [9].

In the context of BRAVE project [10] [11], we target to explore the sub-THz bands mainly between $(90-200 \mathrm{GHz})$ for wireless Terabits per second (Tbps) communication. In this paper, GSM system is analyzed subjected to major sub$\mathrm{THz}$ limitations and RF-impairments which was not considered in the earlier studies. To the best of our knowledge, this is the first paper that considers the performance analysis of GSM systems subjected to PN effects over sub- $\mathrm{THz}$ channels. This performance analysis includes a comparison of GSM systems with different Amplitude-Phase Modulations (APMs) in terms of performance, robustness to PN, computational complexity, Peak-to-Average Power Ratio (PAPR), power consumption. The paper is organized as follows. In section II, GSM system is presented, whereas in section III sub-THz and RF impairments are discussed. Section IV illustrates the results of the APM-GSM comparisons from different perspectives and discusses the feasibility of both systems for ultra-high wireless communication in sub- $\mathrm{THz}$ band. Finally, concluding remarks are given in Section V. 


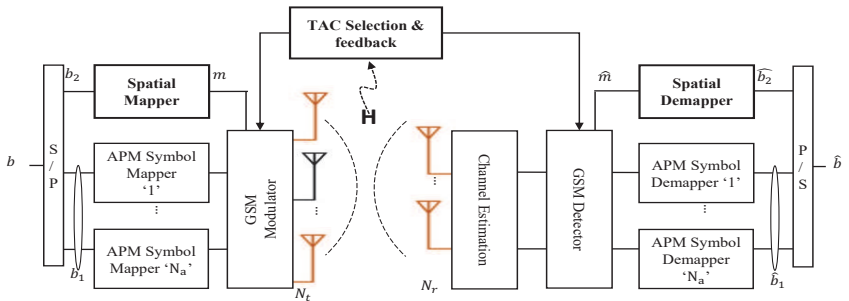

Fig. 1: System model of GSM with TAC selection

The notations used in this paper are as follows. Matrices and vectors are represented by a bold capital and small letters respectively. $\lfloor$.$\rfloor represents the floor function. \|$.$\| stands for$ the Frobenius norm. $(.)^{T}$ is used to denote the transpose of a matrix. $\left(C_{y}^{x}\right)$ represents the binomial coefficient. $\mathcal{C N}\left(\mu, \sigma^{2}\right)$ and $\mathcal{N}\left(\mu, \sigma^{2}\right)$ denotes the complex normal and real normal distribution respectively of a random variable having mean $\mu$ and variance $\sigma^{2}$.

\section{SYSTEM MODEL}

Consider a MIMO GSM system with $N_{r}$ receive antennas (RAs) and $N_{t}$ transmit antennas (TAs) where where only $N_{a}$ TAs are activated at each symbol period to transmit different $M$-ary APM symbols as depicted in Fig. 1. GSM conveys information bits in the signal domain as in MIMO Spatial Multiplexing system, but additional bits are conveyed in the spatial domain by means of IM [1]. Consequently, the possible number of Transmit Antenna Combinations (TACs) is then listed as a combination $C_{N t}^{N a}$ where the number of legitimate TACs is $N_{T A C}=2^{\left.\log _{2}\left(C_{N t}^{N a}\right)\right\rfloor}$, and by indexing the different legitimate TACs, GSM conveys additional information bits by the index of TAC $\left(\log _{2}\left(N_{T A C}\right)\right.$ spatial bits per GSM symbol). Thus, the total number of bits per GSM symbol is given by:

$$
\mathcal{L}_{G S M}=\log _{2}\left(N_{T A C}\right)+N_{a} \log _{2}(M) .
$$

Note that only $N_{T A C}$ are used from $C_{N t}^{N a}$ to keep the spatial bits length as an integer number and the other possibilities are marked as illegal TACs. In the following, we will consider the enhanced GSM system that selects the optimal legitimate TACs sets using the channel side information [3] and the best effort gray spatial-bit mapping [4] (Index-to-Bit mapping) to achieve the optimal GSM performance.

At the receiver end, the signal is represented as

where $\mathbf{H}=\left[\mathbf{h}_{1}, \ldots, \mathbf{h}_{N_{t}} \mathbf{y}=\mathbf{H} \mathbf{x}+\mathbf{n}, \quad\right.$ is the $N_{r} \times N_{t}$ MIMO channel matrix where $\mathbf{h}_{i}$ is the column vector of $N_{r}$ elements, $\mathbf{x}=\left[x_{1}, \ldots, x_{N_{t}}\right]^{T}$ is the transmitted vector that contains $N_{a}$ different APM symbols transmitted through the active TAs according to the index of activated TAC, $\mathbf{n}$ is $N_{r} \times 1$ channel noise vector and its $n_{r}$ elements are complex Gaussian variables, independent and identically distributed (i.i.d.), with zero-mean and variance $\sigma_{n}^{2}$, i.e, $\mathcal{C N}\left(0, \sigma_{n}^{2}\right)$ for $r=1, \ldots, N_{r}$. The energy of the transmitted APM symbols are normalized to unity.

The maximum-likelihood (ML) detector for GSM is able to detect jointly all the $N_{a}$ transmitted APM symbols and the activated TAC by an exhaustive search over all possible transmit vectors:

$$
\widehat{\mathbf{x}}=\arg \min \|\mathbf{y}-\mathbf{H x}\|^{2} .
$$

where $\chi$ contains all possibł $\mathcal{C}^{\prime} \mathrm{GSM}$ vectors that consider both the signal and spatial domain.

\section{SUB-TERAHERTZ CHANNEL AND IMPAIRMENTS}

In this section, the main sub- $\mathrm{THz}$ band impairments and challenges are addressed.

For high frequency broadband systems, the non-linearity of analog components used in Radio-Frequency (RF) front ends gives increased challenges in the modeling of circuits and in anticipating the compensation measures required for performance improvements. The RF front end is composed of all components between the antenna and the digital baseband system of a transceiver, namely mixer or modulator, phase shifter, data converters, and power amplifier (PA). There are many RF challenges at sub-THz bands, especially for low cost implementation:

- Efficiency and achievable transmit output power are low compared to sub-GHz bands, where the maximum achievable output power is in the order of $10 \mathrm{dBm}$.

- Medium to strong Phase noise of the LO that leads to significant degradation in Signal-to-Noise Ratio (SNR) and limits both Bit-Error Rate (BER) performance and throughput rate.

- High power consumption and limited resolution for ultra-high sampling rate for analog-to-digital converters (ADCs) [8]. Note that the total bandwidth available at sub- $\mathrm{THz}$ can be divided into sub-bands of same bandwidth (250 MHz [12], 2.16 GHz [13]). Thus, such a channel arrangement allows to limit the ADC power consumption.

- Non-linearity effect of the Power Amplifier and electronics components.

In addition, the channel at sub- $\mathrm{THz}$ bands suffers from high atmospheric attenuation and very strong obstruction losses (from walls, vegetation, furniture, etc.), and high sensitivity to the environment details (small objects, surface roughness, etc.). In this paper, we focus on short-range indoor scenarios such as kiosk and hot-spot where the atmospheric attenuation is irrelevant.

\subsection{Sub-Terahertz Phase noise model}

It is well known that practical oscillator can never generate a pure sinusoid and the PN increases with the carrier frequency. Thus, the sub-THz communication system should be analyzed under this impairment since neglecting its impact is no more tolerable as in sub-GHz systems.

In general, the analysis of PN impact on MIMO systems is dependent on the RF architecture where there are mainly two LO setups: Distributed Oscillators (DO), Centralized or Common Oscillator (CO). The main difference is that each antenna in the DO setup has its own LO while in the CO 
setup all antennas at the transmitter $\backslash$ receiver are connected to a common LO. Consequently, all parallel streams in $\mathrm{CO}$ has the same PN while the DO suffers from different phase and amplitude distortions.

The PN model is widely investigated where the PN in general is modeled by two main models: Gaussian PN (uncorrelated PN) and Wiener PN (correlated PN). For high-rate communications, the effect of Wiener PN on the system performance is negligible compared to the Gaussian PN [14]. In addition, wiener PN process becomes negligible compared to the Gaussian for wide-band systems where the oscillator corner frequency $f_{c}$ is small compared to the system bandwidth [9]. Thus uncorrelated Gaussian PN model is appropriate for sub- $\mathrm{THz}$ bands. Note that the uncorrelated model remains valid when the following condition is satisfied [9]:

$$
N_{s} f_{c}^{2} T^{2} \leq \frac{\ln (2)}{2 \pi^{2}},
$$

where $N_{s}$ is the number of symbols per frame, $f_{c}$ is the corner frequency of the oscillator and $T$ is the symbol period. It is clear that a careful communication system design can limit the wiener effect by selecting the appropriate $N_{s}$ and $T$.

The received baseband vector of an equivalent $N_{r} \times N_{t}$ MIMO system with phase noise can be expressed as:

$$
\tilde{\mathbf{y}}=\Phi_{r} \mathbf{H} \Phi_{t} \mathbf{x}+\mathbf{n},
$$

where $\Phi_{t}$ and $\Phi_{r}$ are the $N_{t} \times N_{t}$ and $N_{r} \times N_{r}$ diagonal matrices of phase noise from the transmitter and receiver oscillators respectively. These phase noise matrices can be represented as follows:

$$
\begin{gathered}
\Phi_{t}=\operatorname{diag}\left(\left[e^{j \theta_{1}^{T x}}, \ldots, e^{j \theta_{N_{t}}^{T x}}\right]^{T}\right) \\
\Phi_{r}=\operatorname{diag}\left(\left[e^{j \theta_{1}^{R x}}, \ldots, e^{j \theta_{N_{r}}^{R x}}\right]^{T}\right),
\end{gathered}
$$

where $\theta_{i}^{T x}$ and $\theta_{j}^{R x}$ are the phase noise at the $i^{t h}$ TA and $j^{t h}$ RA respectively that can be described in sub- $\mathrm{THz}$ band by a truncated Gaussian distribution $\mathcal{N}\left(0, \sigma_{g}^{2}\right)$ with zero-mean and variance $\sigma_{g}^{2}$ similar to SISO PN model described in [9]. In the DO setup, $\theta_{i}^{T x} \neq \theta_{j}^{T x}$ and $\theta_{i}^{R x} \neq \theta_{j}^{R x}$ for all $i \neq j$. However, in the CO setup, with different oscillators at Tx and Rx sides, $\theta_{i}^{T x}=\theta^{T x}$ for all $i=1, \ldots, N_{t}$ and $\theta_{j}^{R x}=\theta^{R x}$ for all $j=1, \ldots, N_{r}$. Thus, the received signal in the CO setup can be simplified to:

$$
\tilde{\mathbf{y}}=e^{j \theta^{T x}} e^{j \theta^{R x}} \mathbf{H x}+\mathbf{n}
$$

\subsection{Sub-THz MIMO Channel}

A ray-based deterministic channel modeling for sub- $\mathrm{THz}$ Band (mainly between $90-200 \mathrm{GHz}$ ) is presented in [6]. It is worth mentioning that the propagation channel model in [6] considers the material properties, gas attenuation and the impact of furniture that leads to more obstructions along the propagation paths and new scattered paths. In addition, it characterizes the main channel properties such as path loss and delay spread for LOS, NLOS with vegetation and NLOS cases for indoor in-office and outdoor in-street scenarios. In the following, we will focus on the downlink hotspot (or kiosk) indoor scenario where the Access Points
(AP), acting as transmitters, and the Mobile Stations (MS), acting as receivers, are equipped with $N_{t}$ and $N_{r}$ isotropic antennas. Therefore, the MIMO propagation channels are obtained using the simulator for ray-based deterministic channel modeling. The MIMO channels are obtained with different array geometry such as Uniform-Linear-Array (ULA) and Uniform-Rectangular-Array (URA). Note that an antenna element separation of $4 \lambda$, where $\lambda$ is the wavelength, is considered to reduce the effect of spatial correlation [15], and thus enhance MIMO communication.

\section{RESULTS AND DISCUSSIONS}

In this section, the performance of the GSM subjected to sub$\mathrm{THz}$ impairments will be studied using the sub-THz channel model described in previous section. Firstly, we will show the impact of PN in DO setup over MIMO sub-THz channels on GSM systems with different modulation schemes for an indoor ultra-high data rate scenario. The system comparison is performed with different PN levels (low $\sigma_{g}^{2}=0.001$, medium $\sigma_{g}^{2}=0.01$, strong $\sigma_{g}^{2}=0.1$ ) without applying any phase noise mitigation technique. In our comparison, we consider the sub- THz indoor MIMO channels with a separation distance between Mobile Station (MS) and Access Point (AP) going from 2 to 8 meter $(\mathrm{m})$, i.e. an average distance of $5 \mathrm{~m}$. For a fair comparison, the different GSM systems are compared under the same spectral efficiency, which requires the same number of activated TAs $N_{a}$ and the same total number of TAs $N_{t}$ when using a linear modulation schemes as: PSK, QAM, DPSK, PAM. The optimal ML detection is used along with the same number of RAs $N_{r}$. In the following, we will consider different GSM systems with 12 bits per channel use (bpcu) with $N_{t}=10$ and $N_{a}=3$ and one of the following modulations: QPSK, DQPSK, 4PAM. Note DPSK and PAM schemes are considered in our study with MIMO GSM, because they show some robustness to PN in SISO channels.

Fig. 2 shows clearly that QPSK-GSM outperforms DQPSK-GSM and 4PAM-GSM subjected to any PN level.

For example, a gain of 3.4 and $5 \mathrm{~dB}$ is obtained using QPSK-GSM as compared to DQPSK-GSM and 4PAM-GSM systems respectively with $N_{r}=6$ at low PN as shown in Fig. 2.a. However, these values of gain becomes respectively 2.5 and $5.2 \mathrm{~dB}$ when $N_{r}=10$ as shown in Fig. 2.b. Moreover, the performance gain of QPSK-GSM is much higher at medium PN when compared to DQPSK-GSM and 4PAMGSM. Thus, MIMO QPSK-GSM is more robust to PN even when compared to PAM modulation which is not sensitive to PN in SISO mode. Note that these systems, without phase noise mitigation techniques, suffer from a high error floor when subjected to strong PN in sub- $\mathrm{THz}$ channels. The simulation results with URA geometry are shown in Fig. 3, where similar conclusion on the obtained gain can be drawn for low PN with the difference that all systems requires a slightly larger SNR due to higher spatial correlation between antennas. However, only QPSK-GSM maintain the small degradation at medium PN when using URA geometry. 


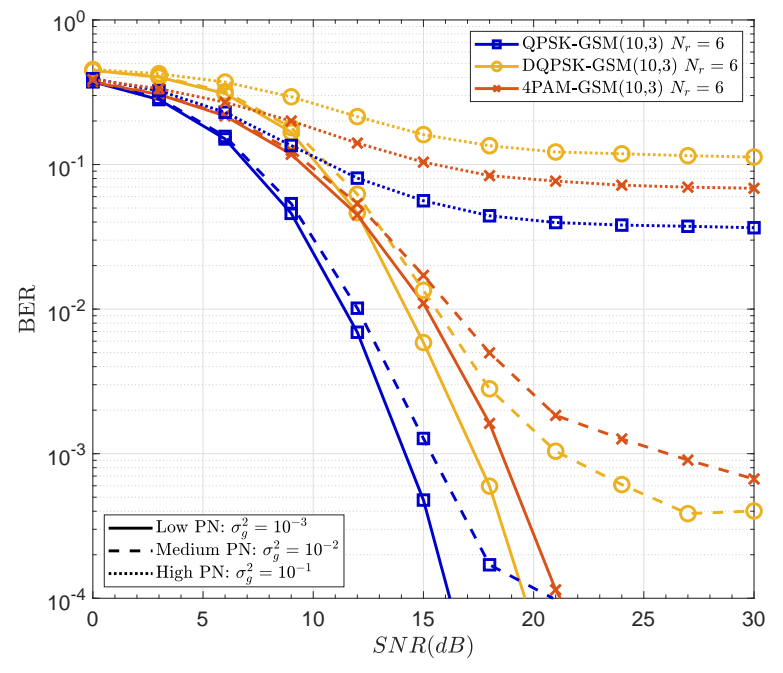

(a)

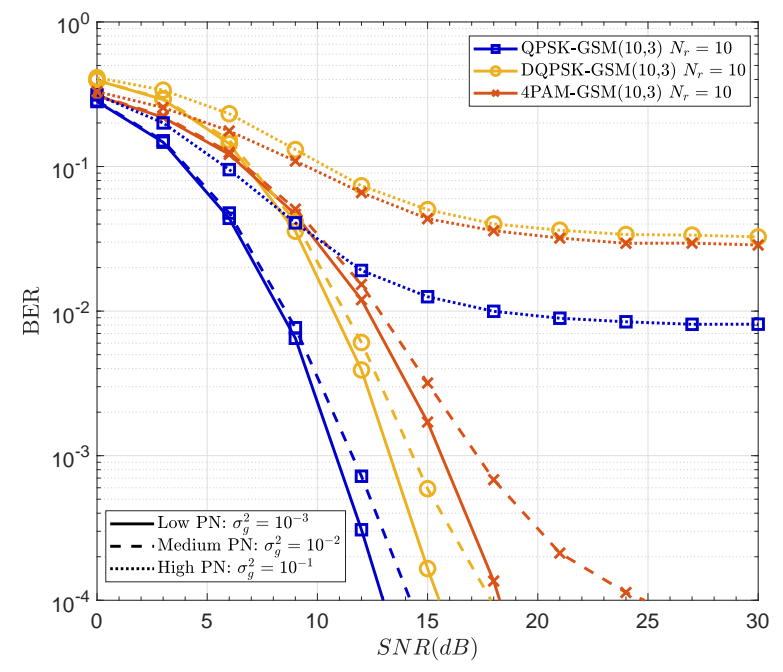

(b)

Fig. 2: BER performance of different APM-GSM systems with sub-THz impairments. The spectral efficiency is 12 bpcu and both systems are subjected to phase noise with different levels using DO setup. The concerned sub-THz indoor MIMO channels are generated using ULA array geometry with $4 \lambda$ antenna separation. The AP-MS distance is $d=[2 m, 8 m]$ with $d_{\text {mean }}=5 m$. (a) $N_{r}=6$, (b) $N_{r}=10$.

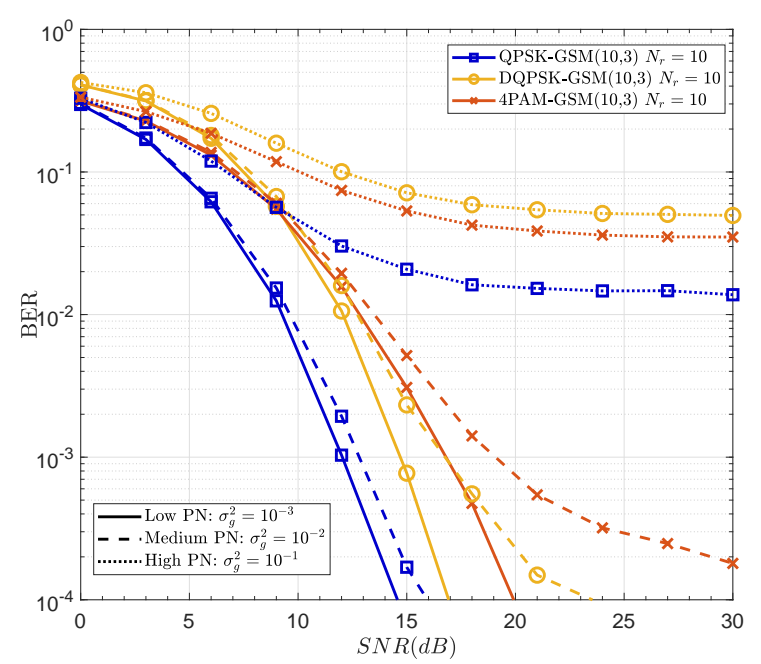

Fig. 3: BER performance of different APM-GSM systems with sub$\mathrm{THz}$ impairments. The spectral efficiency is $12 \mathrm{bpcu}$ and both systems are subjected to phase noise with different levels using DO setup. The concerned sub-THz indoor MIMO channels are generated using URA array geometry with $4 \lambda$ antenna separation and $N_{r}=10$. The AP-MS distance is $d=[2 m, 8 m]$ with $d_{\text {mean }}=$ $5 m$.

Therefore, GSM can survive at low to medium PN in sub$\mathrm{THz}$ channel but more research is required to enhance GSM performance with strong PN channel. We would like to highlight that the performance could be enhanced in PN channel by using PN robust modulations (e.g. spiral QAM [16]), powerful channel coding technique and special detector/equalizer designed specifically for PN channel.

It is worth mentioning that low order modulation schemes are only considered with GSM because they have lower PAPR, lower power consumption and lower sensitivity to PN compared to higher $M$-ary APM schemes (results are not added in this paper due to limited number of pages), and they can be accompanied with low-power high-speed ADCs of few bits resolution. Among the compared low order modulations, QPSK-GSM achieves the lowest power consumption due to the lowest SNR requirements and PAPR. Note that another drawbacks for $M$-ary PAM is that higher $M$-ary PAM suffers from higher PAPR increase and require larger resolution bits for ADCs due to using only the amplitude dimension in the signal domain. Finally, these GSM systems with low order modulation 4-ary APM have similar computational complexity in terms of real multiplication $\backslash$ additions and cost in terms of number of RF chains.

\section{CONCLUSION}

In this paper, the different APM schemes with GSM subjected to realistic sub- $\mathrm{THz}$ impairments in an indoor environment are compared from different perspectives. We emphasized that low order modulation schemes coupled with IM techniques are required to survive with sub- $\mathrm{THz}$ technological limitations, and to achieve ultra-high data rates with acceptable SNR requirement [2]. The simulations results reveal that QPSK-GSM outperforms DQPSK-GSM and 4PAM-GSM for all PN levels in the sub-THz channels using ULA and URA array geometry with a gain reaching $5 \mathrm{~dB}$. Moreover, if the residual PN before detection is in a low to medium level, the presented system QPSK-GSM can be considered as an appropriate solution for indoor ultra-high wireless data rates system in the sub-THz while having a better performance, lower power consumption, lower complexity, higher robustness to $\mathrm{PN}$ and few-bits $\mathrm{ADC}$ resolution requirement compared to other APM-GSM schemes. When a total channel bounding/aggregation of $40 \mathrm{GHz}$ is considered in the $90-200$ $\mathrm{GHz}$ band, a throughput rate of $0.5 \mathrm{Tbps}$ can be achieved. 


\section{REFERENCES}

[1] J. Wang, S. Jia and J. Song, "Generalised Spatial Modulation System with Multiple Active Transmit Antennas and Low Complexity Detection Scheme," in IEEE Trans. Wireless Commun., vol. 11, no. 4, pp. 16051615, April 2012.

[2] M. Saad, F. Bader, J. Palicot, A. C. Al Ghouwayel, H. Hijazi, "Single Carrier with Index Modulation for Low Power Terabit Systems," in the Proc. of the IEEE Wireless Commun. and Netw. Conf. (WCNC), Marrakech, Morocco. Apr. 2019.

[3] L. Xiao et al., "Transmit Antenna Combination Optimization for Generalized Spatial Modulation Systems," in IEEE Access, vol. 6, pp. 41866-41882, 2018.

[4] M. Saad, F. Lteif, A. C. Al Ghouwayel, H. Hijazi, J. Palicot and F. Bader, "Generalized Spatial Modulation in Highly Correlated Channel," IEEE International Symposium on Personal Indoor and Mobile Radio Communications (PIMRC), Istanbul, Turkey, Sept. 2019.

[5] M. Saad, F. Bader, J. Palicot, Y. Corre, G. Gougeon, J-B Doré, "Beyond-5G wireless Tbps Scenarios and Requirements," French funded project-ANR-17-CE250013 BRAVE, Tech. Report BRAVE D1.0, 2018. [Online]. Available: https://hal.archives-ouvertes.fr/hal01947363/document.

[6] G. Gougeon, Y. Corre, M. Aslam. "Ray-based Deterministic Channel Modeling for sub-THz Band," IEEE International Symposium on Personal Indoor and Mobile Radio Communications (PIMRC), Istanbul, Turkey, Sept. 2019.

[7] T. S. Rappaport et al., "Wireless Communications and Applications Above $100 \mathrm{GHz}$ : Opportunities and Challenges for 6G and Beyond," in IEEE Access, vol. 7, pp. 78729-78757, 2019.

[8] B. Murmann, "ADC Performance Survey 1997-2019," [Online]. Available: http://web.stanford.edu/ murmann/adcsurvey.html

[9] S. Bicaïs, J-B. Doré."Phase Noise Model Selection for Sub-THz Communications," IEEE Global Communications Conference 2019, Dec 2019, Waikoloa, United States.

[10] French funded project-ANR-17-CE25-0013, "Back to Single-carrier for beyond-5G communications above 90 GHz-(BRAVE)," [Online]. Available: http://www.brave-beyond5g.com/.

[11] Y. Corre, G. Gougeon, J-B Doré, S. Bicaïs, B. Miscopein, E. Faussurier, M. Saad, J. Palicot, F. Bader.
"Sub-THz Spectrum as Enabler for 6G Wireless Communications up to $1 \mathrm{Tbit} / \mathrm{s}$." in $6 \mathrm{G}$ Wireless Summit, March 2019, Levi Lapland, Finland.

[12] ECC Recommendation (18)01 of 27 April 2018 on "Radio frequency channel/block arrangements for Fixed Service systems operating in the bands 130-134 GHz, 141-148.5 GHz, 151.5$164 \mathrm{GHz}$ and 167-174.8 GHz" [Online]. Available: https://www.ecodocdb.dk/download/a5533f975a92/Rec1801.pdf

[13] IEEE Standard for High Data Rate Wireless MultiMedia Networks-Amendment 2: $100 \mathrm{~Gb} / \mathrm{s}$ Wireless Switched Point-to-Point Physical Layer," in IEEE Std 802.15.3d-2017 (Amendment to IEEE Std 802.15.32016 as amended by IEEE Std 802.15.3e-2017), vol., no., pp.1-55, 18 Oct. 2017

[14] M. R. Khanzadi, D. Kuylenstierna, A. Panahi, T. Eriksson, and H. Zirath, "Calculation of the performance of communication systems from measured oscillator phase noise," IEEE Trans. on Circuits and Systems I: Regular Papers, vol. 61, no. 5, pp. 1553-1565, May 2014.

[15] A. Chatterjee, S. Chatterjee and S. S. Das, "Evaluation of spatial correlation and its effect on channel capacity of uniform planar antenna array," 2017 Twenty-third National Conference on Communications (NCC), Chennai, 2017, pp. 1-6.

[16] A. Ugolini, A. Piemontese and T. Eriksson, "Spiral Constellations for Phase Noise Channels," in IEEE Trans. on Commun., vol. 67, no. 11, pp. 7799-7810, Nov. 2019. 